TRANSACTIONS OF THE

AMERICAN MATHEMATICAL SOCIETY

Volume 363, Number 6, June 2011, Pages 2891-2906

S 0002-9947(2011)05121-4

Article electronically published on January 7, 2011

\title{
GROUP ACTIONS ON GEODESIC PTOLEMY SPACES
}

\author{
THOMAS FOERTSCH AND VIKTOR SCHROEDER
}

\begin{abstract}
In this paper we study geodesic Ptolemy metric spaces $X$ which allow proper and cocompact isometric actions of crystallographic or, more generally, virtual polycyclic groups. We show that $X$ is equivariantly roughly isometric to a Euclidean space.
\end{abstract}

\section{INTRODUCTION}

1.1. Statement of results. A metric space $X$ is called a Ptolemy metric space if the inequality

$$
|x y||u v| \leq|x u||y v|+|x v||y u|
$$

is satisfied for all $x, y, u, v \in X$.

In this paper we study subgroups $\Gamma \subset \operatorname{ISO}(X)$ of the isometry group of geodesic Ptolemy spaces $X$, which act properly and cocompactly on $X$. Our motivation to study these spaces is described in Subsection 1.3.

We adopt the notation of $[\mathrm{BH}]$ (p. 131) and call an action proper if for every $x \in X$ there is a number $r>0$ such that the set $\{\gamma \in \Gamma|| x \gamma(x) \mid<r\}$ is finite. An action is cocompact if there is a compact subset $K \subset X$, such that $X=\Gamma K$. Since $X$ allows a proper and cocompact action by isometries, $X$ is complete and, since $X$ is geodesic, it is indeed a proper metric space, i.e. closed distance balls are compact (see [BH], p. 132, 8.4(1)).

The fact that $X$ is a proper metric space is used essentially in our paper.

We recall that a group $\Gamma$ is called crystallographic if, for some $n \in \mathbb{N}, \Gamma$ is isomorphic (as an abstract group) to a discrete, cocompact subgroup of the isometry group $\operatorname{ISO}\left(\mathbb{R}^{n}\right)$. The number $n$ is then called the rank of $\Gamma$. A map $\varphi: X \rightarrow Y$ between metric spaces is called roughly isometric if there exists a constant $A \geq 0$ such that for all $x, x^{\prime} \in X$ we have

$$
\left|x x^{\prime}\right|-A \leq\left|\varphi(x) \varphi\left(x^{\prime}\right)\right| \leq\left|x x^{\prime}\right|+A .
$$

If, in addition, for all $y \in Y$ there exists $x \in X$ with $|y \varphi(x)| \leq A$, we call $\varphi$ a rough isometry.

Theorem 1.1. Let $X$ be a geodesic Ptolemy metric space and let $\Gamma \subset \operatorname{ISO}(X)$ be a crystallographic group of rank $n$ acting properly and cocompactly on $X$. Then there exists a continuous rough isometry $\varphi: X \rightarrow \mathbb{R}^{n}$, which is equivariant with respect

Received by the editors December 16, 2008.

2010 Mathematics Subject Classification. Primary 51F99, 53C23.

Both authors were supported by the Swiss National Science Foundation, grant 200021-115919. 
to the group operation, i.e. $\varphi(\gamma x)=\gamma \varphi(x)$, where $\Gamma$ acts on $\mathbb{R}^{n}$ by isometries with compact quotient.

In the case where $X$ is a topological manifold we can say more.

Theorem 1.2. Let $X$ be a geodesic Ptolemy metric space which is in addition a topological manifold. Assume that $\Gamma \subset \operatorname{ISO}(X)$ is a crystallographic group of rank $n$ which acts properly and cocompactly on $X$. Then there actually exists an equivariant isometry $\varphi: X \rightarrow \mathbb{R}^{n}$.

The methods of the proof in connection with the results in [CS also give the following general structure result for abelian groups operating on Ptolemy spaces:

Theorem 1.3. Let $X$ be a geodesic Ptolemy metric space and let $\Gamma \subset \operatorname{ISO}(X)$ act properly and cocompactly on $X$. Then every free abelian subgroup of $\Gamma$ is straight in $\Gamma$.

To define the notion of straightness we recall the word norm ||$_{\Gamma}$ of a finitely generated group. Let $\left\{\gamma_{1}, \ldots, \gamma_{k}\right\}$ be a set of generators of $\Gamma$. Then for $\gamma \in \Gamma,|\gamma|_{\Gamma}$ is defined as the length of the shortest word in the $\gamma_{i}$ and $\gamma_{i}^{-1}$ representing $\gamma$. This norm depends on the choice of generators. However, as it is easy to see, different sets of generators give rise to equivalent norms. That is, if ||$_{\Gamma}^{1}$ and ||$_{\Gamma}^{2}$ are such norms, then there exists a constant $c \geq 1$ such that $(1 / c)|\gamma|_{\Gamma}^{1} \leq|\gamma|_{\Gamma}^{2} \leq c|\gamma|_{\Gamma}^{1}$ for all $\gamma \in \Gamma$. A finitely generated subgroup $\Gamma_{0} \subset \Gamma$ is called straight in $\Gamma$ if ||$_{\Gamma_{0}}$ and ||$_{\Gamma}$ are equivalent norms on $\Gamma_{0}$. This notion is independent of the choice of generators on $\Gamma_{0}$ and $\Gamma$.

As a consequence of Theorem 1.3 we can generalize our result to the action of virtual polycyclic groups. A group is called polycyclic if it admits a finite chain of subgroups $G_{0} \subset G_{1} \subset \cdots \subset G_{l}$, such that $G_{0}$ is the trivial subgroup and $G_{l}=G$, such that $G_{i} / G_{i-1}$ is cyclic. Equivalently a group is polycyclic if it is solvable and every subgroup is finitely generated. A group is called virtual polycyclic if it contains a polycyclic subgroup of finite index. In particular crystallographic groups are virtual polycyclic.

Corollary 1.4. Theorem 1.1 and Theorem 1.2 also hold if $\Gamma \subset \operatorname{ISO}(X)$ is assumed to be virtual polycyclic. In particular if a virtual polycyclic group acts properly and cocompactly on a geodesic Ptolemy space, then there exists a homomorphism $\Gamma \rightarrow \operatorname{ISO}\left(\mathbb{R}^{n}\right)$ with finite kernel such that the image is a crystallographic group.

At the end of the introduction we state a result, which we need in the proof and which is of independent interest; see Section 2 .

Theorem 1.5. A proper, geodesic Ptolemy metric space is strictly distance convex.

1.2. Outline of the main ideas, Ptolemy versus CAT(0). All the above results are well known if the space $X$ is a $\operatorname{CAT}(0)$-space. A CAT(0)-space is Ptolemy (see [FLS]). It is an open question if proper Ptolemy metric spaces are CAT(0) (we remark that there are nonlocally compact Ptolemy spaces which are not CAT(0); see [FLS]). To be very concrete, we do not even know whether the flat strip theorem of CAT(0)-spaces (see e.g. $[\mathrm{BH}]$, p. 182) holds for Ptolemy metric spaces: Assume that $d$ is a distance function on the set $X=\mathbb{R} \times[0,1]$, such that $(X, d)$ is a geodesic Ptolemy space, the topology on $(X, d)$ coincides with the standard 
topology, the curves $t \mapsto(t, i)$ are geodesics parameterized by arclength for $i=0,1$ and $d((t, 0),(t, 1)) \leq A$ for some constant $A$. Question: Is $(X, d)$ isometric to a flat strip $\mathbb{R} \times[0, D]$ ? We do not know the answer even under the additional assumption that the translation map $T: X \rightarrow X, T(t, s)=(t+1, s)$ is an isometry. In the case of $\operatorname{CAT}(0)$-spaces the flat strip theorem follows from the fact that the distance function $t \mapsto d\left(c(t), c^{\prime}(t)\right)$ is convex for geodesics $c, c^{\prime}:[a, b] \rightarrow X$. We also remark that the convexity of the function $t \mapsto d\left(c(t), c^{\prime}(t)\right)$ is the essential tool to obtain the analogous results of Theorem 1.1 and Theorem 1.2 in the CAT(0)-case. Since we do not have this convexity, our proof in the Ptolemy case is very different.

We outline our main ideas: Our proof relies on a weaker form of convexity. In geodesic Ptolemy metric spaces the distance function $d(p,$.$) to a point p \in X$ is convex. As a consequence Busemann functions are also convex. We show (in the setting of Theorem (1.2) that the Busemann function of an axis of an element $\alpha \in \Gamma^{*} \subset \Gamma$ (where $\Gamma^{*}$ is the maximal abelian subgroup of $\Gamma$ ) is indeed an affine function. We can show that there are enough affine functions to separate points. Thus we can apply the main theorem of HL to obtain that $X$ is isometric to a convex subset of a normed vector space. Finally we have to use the Theorem of Schoenberg $[\mathrm{Sch}$ to see that $X$ is isometric to a Euclidean space. In the setting of Theorem 1.1 we need suitable modifications.

We should mention that the result and parts of its proof have some analogy to the "Hopf conjecture" and its proof in [BuI]. Compare in this context also $[\mathrm{Bu}]$ and the discussion in G], pp. $43 \mathrm{ff}$.

1.3. Motivation and outlook. At the end of this introduction we give an idea why we investigate geodesic Ptolemy spaces. Our motivation was the study of the boundary of CAT $(-1)$-spaces. On the ideal boundary $\partial_{\infty} Y$ of a CAT $(-1)$-space $Y$, Bourdon [B] introduced a distance function $\rho_{o}$, depending on a point $o \in Y$. A similar metric $\rho_{\omega}$ on $\partial_{\infty} Y \backslash\{\omega\}$ was introduced by Hamenstädt [Ha], where the metric depends on a point $\omega \in \partial_{\infty} Y$ and a Busemann function for this point. The metrics $\rho_{o}, \rho_{\omega}$ are all Möbius equivalent. For a detailed description compare [FS].

Let us describe the elements of Möbius geometry on metric spaces. Let $X$ be a metric space, where $|x y|$ is the distance between $x$ and $y$. To a quadruple $(x, y, z, w) \in X^{4} \backslash D$ (where $D$ is the diagonal of points where one entry appears 3 or 4 times), we associate the cross ratio triple

$$
\operatorname{crt}(x, y, z, w)=(|x y||z w|:|x z||y w|:|x w||y z|) \in \mathbb{R} P^{2} .
$$

Similar to the classical cross ratio, there are six reasonable definitions obtained by permuting the entries. A map $f: X \rightarrow Y$ is called a Möbius map if it is injective and for all quadruples from $X^{4} \backslash D$ we have $\operatorname{crt}(f(x), f(y), f(z), f(w))=\operatorname{crt}(x, y, z, w)$. This definition is equivalent to the usual definition using the classical cross ratio. Let $\Delta \subset \mathbb{R} P^{2}$ be the subset of points $(a: b: c)$ such that the three numbers $|a|,|b|,|c|$ satisfy the triangle inequality. Then $X$ is a Ptolemy metric space if and only if for all allowed quadruples, $\operatorname{crt}(x, y, z, w) \in \Delta$. Therefore the Ptolemy condition is in a natural way a Möbius invariant condition. In a certain sense the Ptolemy condition is the natural condition for involutive geometry: note that a metric space $X$ is Ptolemy if and only if for all points $x \in X$ the map $\rho_{x}: X \backslash\{x\} \times X \backslash\{x\} \rightarrow \mathbb{R}$, $\rho_{x}(y, z)=\frac{|y z|}{|y x||z x|}$ is a metric (see also [BFW]).

In [FS] we made the observation that the Bourdon and Hamenstädt metrics on $\partial_{\infty} Y$ are Ptolemy for CAT(-1)-spaces $Y$ and the isometries of $Y$ extend to Möbius 
maps on $\partial_{\infty} Y$. In Ptolemy metric spaces one can naturally define the notion of a circle (in the generalized sense, i.e. a circle or a line). Recall that the classical Ptolemy theorem states that four points $x, y, z, w$ (in the Euclidean plane or more generally in $\mathbb{R}^{n}$ ) lie on a circle (or a line) if and only if $\operatorname{crt}(x, y, z, w) \in \partial \Delta$, where $\partial \Delta$ is the set of points $(a: b: c)$ with equality in the triangle inequality. Thus let us define

Definition 1.6. Let $X$ be a Ptolemy space. A circle in $X$ is a continuous map $f: S^{1} \rightarrow X$ such that for all $x, y, z, w \in S^{1}$ we have equality in the Ptolemy inequality, i.e. $\operatorname{crt}(f(x), f(y), f(z), f(w)) \in \partial \Delta$.

It then turns out that for points $x, y, z, w \in S^{1}$, which are ordered cyclically on $S^{1}$ (i.e. $y$ and $w$ lie in different components of $S^{1} \backslash\{x, z\}$ ), we have an equality of the form

$$
|f(x) f(y)||f(z) f(w)|+|f(y) f(z)||f(w) f(x)|=|f(x) f(z)||f(w) f(y)| .
$$

Note that this notion of a circle is obviously Möbius invariant and thus circles are preserved by Möbius maps.

Now one can reformulate a result of Bourdon (see [B]) in the following way: Let $Y$ be a CAT(-1)-space and consider $\partial_{\infty} Y$ as a Ptolemy space with some Bourdon or Hamenstädt metric. Let $f: S^{1} \rightarrow \partial_{\infty} Y$ be a circle. Then there exists a totally geodesic subspace $Y_{0} \subset Y$ isometric to $\mathbb{H}^{2}$ such that $\partial_{\infty} Y_{0}$ is the image of $f$. Indeed the circles in $\partial_{\infty} Y$ correspond $1-1$ to the boundaries of isometrically embedded hyperbolic planes in $Y$.

This is one motivation for studying Ptolemy spaces with many circles. If we look to our definition of a circle, a circle through infinity should just be a proper map $f: \mathbb{R} \rightarrow X$ such that for all allowed quadruples $x, y, z, w \in \mathbb{R}$ we have $\operatorname{crt}(f(x), f(y), f(z), f(w)) \in \partial \Delta$. For arbitrary but fixed $x, y, z$ and $w \rightarrow \infty$ this implies that $(|f(x) f(y)|:|f(x) f(z)|:|f(y) f(z)|) \in \partial \Delta$. Equivalently, this condition says that $f: \mathbb{R} \rightarrow X$ is a geodesic line (not necessarily parameterized proportionally to arclength). From this viewpoint it is also natural to study Ptolemy spaces with many lines.

\section{Convexity}

Let $X$ be a metric space. By $|x y|$ we denote the distance between points $x, y \in X$. Unless otherwise stated, we will parameterize geodesics proportionally to arclength. Thus a geodesic in $X$ is a map $c: I \rightarrow X$ with $|c(t) c(s)|=\lambda|t-s|$ for all $s, t \in I$ and some constant $\lambda \geq 0$. A metric space is called geodesic if every pair of points can be joined by a geodesic. In the sequel $X$ will always denote a geodesic metric space. For $x, y \in X$ we denote by $m(x, y)=\left\{z \in X|| x z|=| z y\left|=\frac{1}{2}\right| x y \mid\right\}$ the set of midpoints of $x$ and $y$. A subset $C \subset X$ is convex if for $x, y \in C$ also $m(x, y) \subset C$.

A function $f: X \rightarrow \mathbb{R}$ is convex (resp. affine) if for all geodesics $c: I \rightarrow X$ the map $f \circ c: I \rightarrow \mathbb{R}$ is convex (resp. affine).

The space $X$ is called distance convex if for all $p \in X$ the distance function $d_{p}=|\cdot p|$ to the point $p$ is convex. It is called strictly distance convex if the functions $t \mapsto\left(d_{p} \circ c\right)(t)$ are strictly convex whenever $c: I \rightarrow X$ is a geodesic with $|c(t) c(s)|>|| p c(t)|-| p c(s)||$ for all $s, t \in I$, i.e., with neither $c(t)$ nor $c(s)$ being on a geodesic from $p$ to the other. This definition is natural, since the restriction of $d_{p}$ 
to a geodesic segment containing $p$ is never strictly convex. The Ptolemy property easily implies (see [FLS]):

Lemma 2.1. A geodesic, Ptolemy metric space is distance convex.

As a consequence, we obtain that for Ptolemy metric spaces, local geodesics are geodesics. Here we call a map $c: I \rightarrow X$ a local geodesic if for all $t \in I$ there exists a neighborhood $t \in I^{\prime} \subset I$ such that $c_{\mid I^{\prime}}$ is a geodesic.

Lemma 2.2. If $X$ is distance convex, then every local geodesic is globally minimizing.

Proof. Assume the contrary. Then there is a local geodesic $c:[0, b] \rightarrow X$ such that there exists $a \in(0, b)$ such that for $p=c(0)$ we have $|p c(a-\varepsilon)|=a-\varepsilon$ for all $\varepsilon \geq 0$ but $|p c(a+\varepsilon)|<a+\varepsilon$ for $\varepsilon>0$. The distance convexity applied to $d_{p}$ and the minimizing geodesic $c_{\mid[a-\varepsilon, a+\varepsilon]}$ implies that $|p c(a)|<a$, a contradiction.

In [FLS] we gave examples of Ptolemy metric spaces which are not strictly distance convex. However, if the space is proper, then the situation is completely different.

Theorem 1.5. A proper, geodesic Ptolemy metric space is strictly distance convex.

Before we prove this result, we derive some consequences. The first corollary has already been proved in [FLS.

Corollary 2.3 (see also [FLS]). Let $X$ be a proper, geodesic Ptolemy space. Then for $x, y \in X$ there exists a unique midpoint $m(x, y) \in X$. The midpoint function $m: X \times X \rightarrow X$ is continuous.

Proof. Assume that there are midpoints $m_{1}, m_{2} \in m(x, y)$. Let $m$ be a midpoint of $m_{1}$ and $m_{2}$. If $m_{1} \neq m_{2}$, then the strict distance convexity according to Theorem 1.5 implies $|x m|<\left|x m_{i}\right|=\frac{1}{2}|x y|$, and $|y m|<\left|y m_{i}\right|=\frac{1}{2}|x y|$, which is a contradiction. The uniqueness of the midpoint function together with the properness easily implies the continuity of $m$.

Corollary 2.4. Let $X$ be a proper, geodesic Ptolemy space, and let $K \subset X$ be a compact subset. Then there exists a unique closed ball $B_{r}(p)$ of minimal radius such that $K \subset B_{r}(p)$.

Proof. The existence of such a ball of minimal radius is clear. We have to prove the uniqueness. Assume that $K \subset B_{r}\left(p_{1}\right) \cap B_{r}\left(p_{2}\right)$, with $p_{1} \neq p_{2}$. Let $m$ be the midpoint of $p_{1}$ and $p_{2}$. If $q \in K$, then $\left|q p_{i}\right| \leq r$ and the strict convexity of the distance function implies $|q m|<r$. By compactness, $K \subset B_{r^{\prime}}(m)$ for some $r^{\prime}<r$, contradicting the minimality of $r$.

Corollary 2.5. Let $X$ be a proper, geodesic Ptolemy space, and let $A \subset X$ be a closed and convex subset. Then there exists a continuous projection $\pi: X \rightarrow A$.

Proof. The strict convexity of the distance function easily implies, with a similar argument as in the proof above, that for given $x \in X$, there exists a unique $a \in A$, with $|a x|$ minimal. One easily checks that this projection is continuous.

Remark 2.6. For CAT(0)-spaces this projection is always 1-Lipschitz. We do not know if $\pi$ is 1-Lipschitz for general proper geodesic Ptolemy spaces. 
The strict convexity of the distance function together with the properness implies easily (cf. Corollary 2.3)

Corollary 2.7. Let $X$ be a proper, geodesic Ptolemy space and let $x, y \in X$. Then there exists a unique geodesic $c_{x y}:[0,1] \rightarrow X$ from $x$ to $y$ and the map $X \times X \times$ $[0,1] \rightarrow X,(x, y, t) \mapsto c_{x y}(t)$ is continuous.

As a consequence $X$ is contractible. Indeed for the choice of a basepoint $o \in X$ the map $\rho: X \times[0,1] \rightarrow X, \rho_{t}(x)=c_{o x}(t)$ is a contraction.

Corollary 2.8. Let $X$ be a complete, geodesic Ptolemy space. If $X$ is a topological manifold, then every geodesic is extendable. Thus for every geodesic segment $c$ : $I \rightarrow X$ there exists a geodesic $\bar{c}: \mathbb{R} \rightarrow X$ with $\bar{c}_{\mid I}=c$.

Proof. Let $c: I \rightarrow X$ be a geodesic defined on some interval $I \subset \mathbb{R}$, which cannot be extended. We have to show that $I=\mathbb{R}$. If $c$ is a constant map, then it can be extended trivially. Thus $I$ consists of more than one point and $c$ is not a constant geodesic. Since $X$ is complete, we see that $I$ is (after obvious reparameterization) of the form $[0, a]$ with $a>0$ or of the form $(-\infty, a]$ for some $a>0$. In both cases the geodesic $c_{[[0, a]}:[0, a] \rightarrow X$ cannot be extended over its endpoint $c(a)$, since otherwise $c$ could also be extended by Lemma 2.2. Let $o=c(0)$ and $x=c(a)$. The nonextendability of $c_{[0, a]}$ implies that for every point $y \in X \backslash\{x\}$, the point $x$ is not contained in $c_{o y}([0,1])$. Thus $\rho$ also defines a retraction $\rho_{\mid X \backslash\{x\}}: X \backslash\{x\} \times[0,1] \rightarrow$ $X \backslash\{x\}$, and hence $X$ as well as $X \backslash\{x\}$ are contractible. This is in contradiction to the fact that $H_{n}(X,\{x\}, \mathbb{Z})=\mathbb{Z}$, since $X$ is a manifold.

We now prove Theorem 1.5. We start with a simple observation.

Lemma 2.9. If $\alpha, \beta, a, b$ are real numbers with $\alpha+\beta, a, b \geq 0$, then

$$
\alpha b+\beta a \geq(\alpha+\beta) \min \{a, b\}-|a-b| \min \{|\alpha|,|\beta|\} .
$$

Proof. We assume without loss of generality that $a \leq b$. Then $b=a+c$, where $c=|a-b| \geq 0$. We compute $\alpha b+\beta a=(\alpha+\beta) a+c \alpha$. If $\alpha \geq 0$, then our estimate holds trivially. If $\alpha<0$, then the assumption implies that $|\alpha|=\min \{|\alpha|,|\beta|\}$ and $c \alpha=-|a-b| \min \{|\alpha|,|\beta|\}$.

Proof of Theorem 1.5. Note that in order to prove Theorem 1.5] it suffices to prove the

Claim. Let $X$ be a proper, geodesic, Ptolemy metric space. Let further $p, x, y \in X$ be points with

$$
|| p x|-| p y||<|x y|
$$

or equivalently, neither $x$ nor $y$ is on a geodesic from $p$ to the other.

Then there exists a midpoint $m$ of $x$ and $y$ such that $|p m|<\frac{1}{2}[|p x|+|p y|]$.

Indeed, since a geodesic Ptolemy metric space is distance convex by Lemma 2.1. the claim implies the uniqueness of midpoints and therefore the validity of Theorem 1.5 .

In order to prove the claim, we set

$$
\eta:=\frac{\| p x|-| p y||}{|x y|}
$$


Thus, $0 \leq \eta<1$ by assumption (41). Let $s:=\frac{1}{2}[|p x|+|p y|]$ and let $\tau:=\frac{|p x|}{s}$ as well as $\rho:=\frac{|p y|}{s}$. Thus, $0<\tau, \rho<2$ and $\tau+\rho=2$.

We first make the additional assumption that $\tau$ and $\rho$ are very close together; i.e., both are very close to 1 . Namely we assume that

$$
\min \left\{\frac{1}{\tau}, \frac{1}{\rho}\right\}-\eta \frac{\min \{\tau, \rho\}}{\tau \rho} \geq \frac{1}{2}(1-\eta) .
$$

Clearly this assumption holds for $\rho=1=\tau$ (and hence $\eta=0$ ), and at first reading one might as well consider this special case. We will now prove the claim under the Assumption (5). Later we will reduce the general case to this special situation.

Choose geodesics $\gamma_{p x}, \gamma_{p y}:[0, s] \longrightarrow X$ connecting $p$ to $x$ and $y$, parameterized proportionally to arclength. For every $t \in[0, s]$, let $\sigma_{s-t}:[0,2] \longrightarrow X$ be a geodesic connecting $\gamma_{p x}(s-t)$ to $\gamma_{p y}(s-t)$, parameterized proportionally to arclength. Since $X$ is proper, there exists a sequence $t_{n} \in(0, s), t_{n} \longrightarrow 0$, such that $\sigma_{s-t_{n}}(1) \longrightarrow m$, where $m$ is some midpoint of $x$ and $y$.

The distance convexity of geodesic, Ptolemy metric spaces implies $|p m| \leq \frac{1}{2}[|p x|+$ $|p y|]$. To prove our claim, we assume equality and will finally reach a contradiction.

Thus we assume $|p m|=\frac{1}{2}[|p x|+|p y|]=s$.

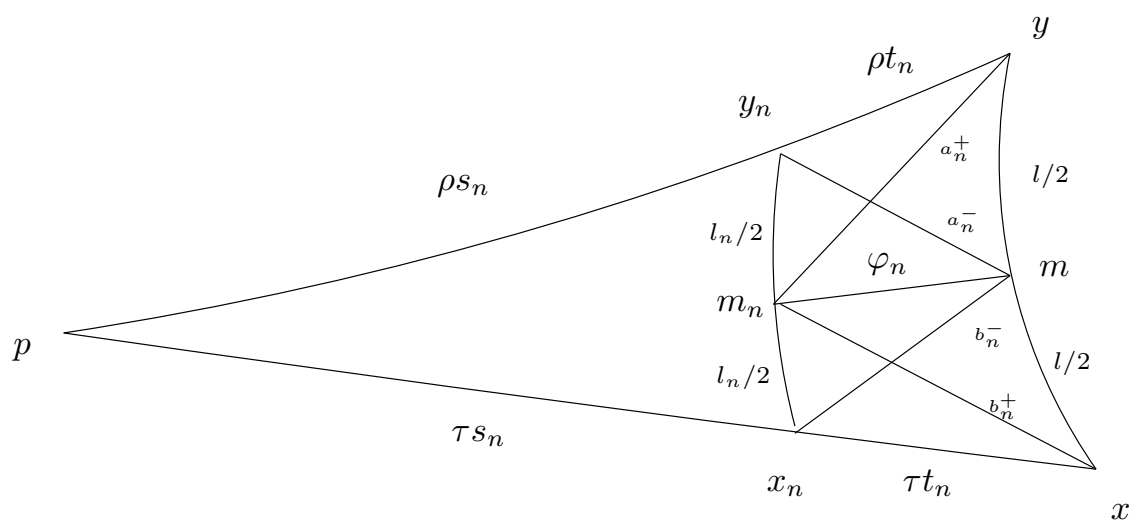

Figure 1. The figure visualizes the notation in the proof of Theorem 1.5 .

We set

$$
\begin{array}{lll}
s_{n}:=s-t_{n}, & x_{n}:=\gamma_{p x}\left(s_{n}\right), & y_{n}:=\gamma_{p y}\left(s_{n}\right) \\
m_{n}:=\sigma_{s_{n}}(1), & l_{n}:=\left|x_{n} y_{n}\right|, & l:=|x y| \\
b_{n}^{-}:=\left|x_{n} m\right|, & a_{n}^{-}:=\left|y_{n} m\right|, & b_{n}^{+}:=\left|x m_{n}\right| \\
a_{n}^{+}:=\left|y m_{n}\right| & \text { and } & \varphi_{n}:=\left|m_{n} m\right|
\end{array}
$$

Since $m_{n} \rightarrow m$ we have $\varphi_{n} \stackrel{n \rightarrow \infty}{\longrightarrow} 0$.

By a simple triangle inequality, the Ptolemy inequality for $\left\{p, x_{n}, m, y_{n}\right\}$ and the sum of the Ptolemy inequalities for $\left\{x_{n}, x, m, m_{n}\right\}$ and $\left\{m_{n}, m, y, y_{n}\right\}$, we obtain 
the inequalities

$$
\begin{aligned}
a_{n}^{+}+b_{n}^{+} & \geq l, \\
(I I) \quad a_{n}^{-} \tau s_{n}+b_{n}^{-} \rho s_{n} & \geq l_{n}\left(s_{n}+t_{n}\right) \text { as well as } \\
\text { (III }) \quad a_{n}^{+} a_{n}^{-}+b_{n}^{+} b_{n}^{-} & \leq \frac{l l_{n}}{2}+2 t_{n} \varphi_{n} .
\end{aligned}
$$

Now the distance convexity (Lemma 2.1) yields

$$
b_{n}^{-} \leq \frac{1}{2}\left(\tau t_{n}+\left|x_{n} y\right|\right) \leq \frac{1}{2}\left(\tau t_{n}+\rho t_{n}+l_{n}\right)=\frac{1}{2} l_{n}+t_{n}
$$

and similarly $a_{n}^{-} \leq \frac{1}{2} l_{n}+t_{n}$. These inequalities together with the triangle inequality $a_{n}^{-}+b_{n}^{-} \geq l_{n}$ also yield $b_{n}^{-} \geq \frac{1}{2} l_{n}-t_{n}$ as well as $a_{n}^{-} \geq \frac{1}{2} l_{n}-t_{n}$. Hence we obtain $\left|a_{n}^{-}-\frac{l_{n}}{2}\right| \leq t_{n}$ and $\left|b_{n}^{-}-\frac{l_{n}}{2}\right| \leq t_{n}$.

In the same way, we deduce $\left|a_{n}^{+}-\frac{l}{2}\right| \leq t_{n}$ and $\left|b_{n}^{+}-\frac{l}{2}\right| \leq t_{n}$.

Thus, passing to appropiate subsequences, we find $A^{-}, B^{-}, A^{+}, B^{+} \in(-2,2)$ such that

$$
\begin{array}{ll}
a_{n}^{-}=\frac{l_{n}}{2}+\frac{A^{-}}{\tau} t_{n}+o\left(t_{n}\right), & b_{n}^{-}=\frac{l_{n}}{2}+\frac{B^{-}}{\rho} t_{n}+o\left(t_{n}\right), \\
a_{n}^{+}=\frac{l}{2}+\frac{A^{+}}{\tau} t_{n}+o\left(t_{n}\right), & b_{n}^{+}=\frac{l}{2}+\frac{B^{+}}{\rho} t_{n}+o\left(t_{n}\right)
\end{array}
$$

and

$$
\left|\frac{A^{-}}{\tau}\right|,\left|\frac{B^{-}}{\rho}\right|,\left|\frac{A^{+}}{\tau}\right|,\left|\frac{B^{+}}{\rho}\right| \leq 1 .
$$

The inequalities $(I),(I I)$ and $(I I I)$ above now yield

$$
\begin{aligned}
& \left(I^{\prime}\right) \\
& \frac{A^{+}}{\tau}+\frac{B^{+}}{\rho} \geq 0, \\
& A^{-}+B^{-} \geq \frac{l}{s} \text { as well as } \\
& \left(I I I^{\prime}\right) \quad \frac{A^{-}}{\tau}+\frac{A^{+}}{\tau}+\frac{B^{-}}{\rho}+\frac{B^{+}}{\rho} \leq 0 .
\end{aligned}
$$

But Lemma 2.9, the inequalities (6), inequality $\left(I I^{\prime}\right),|\tau-\rho|=\frac{l}{s} \eta$ and inequality (5) also yield

$$
\begin{aligned}
\frac{A^{-}}{\tau}+\frac{B^{-}}{\rho} & \geq\left(A^{-}+B^{-}\right) \min \left\{\frac{1}{\tau}, \frac{1}{\rho}\right\}-\left|\frac{1}{\rho}-\frac{1}{\tau}\right| \min \left\{\left|A^{-}\right|,\left|B^{-}\right|\right\} \\
& \geq \frac{l}{s} \min \left\{\frac{1}{\tau}, \frac{1}{\rho}\right\}-\frac{|\tau-\rho|}{\rho \tau} \min \{\tau, \rho\} \\
& \geq \frac{l}{s} \frac{1}{2}(1-\eta)>0,
\end{aligned}
$$

which contradicts the simultaneous validity of the inequalities $\left(I^{\prime}\right)$ and $\left(I I I^{\prime}\right)$.

Finally we have to get rid of the assumption that $\rho$ and $\tau$ are close together. Consider some arbitrary $p, y_{0}, y_{2}$ with $\eta:=\frac{|| p y_{0}|-| p y_{2}||}{\left|y_{0} y_{2}\right|}<1$. Consider a geodesic $[0,2] \rightarrow X, t \mapsto y_{t}$ from $y_{0}$ to $y_{2}$ parameterized proportionally to arclength. If $\left|p y_{1}\right|<s_{0}=\frac{1}{2}\left(\left|p y_{0}\right|+\left|p y_{2}\right|\right)$, we are done. Thus we assume $\left|p y_{1}\right|=s_{0}$, which then implies by Lemma 2.1 that $t \mapsto\left|p y_{t}\right|$ is affine. As a consequence we see that

$$
\frac{|| p y_{1-t}|-| p y_{1+t}||}{\left|y_{1-t} y_{1+t}\right|}=\eta
$$


is constant. Thus if we take as a new triple the points $p, y_{1-t}, y_{1+t}$ for some $t>0$, then the corresponding $\eta$ is the same as for the original triple. Now we can choose $t>0$ sufficiently small, such that for $y_{0}^{\prime}:=y_{1-t}, y_{2}^{\prime}:=y_{1+t}$ and $\rho^{\prime} s_{0}=\left|p y_{0}^{\prime}\right|$, $\tau^{\prime} s_{0}=\left|p y_{2}^{\prime}\right|$ the estimate (5) holds (this is possible, since $\eta$ is fixed and $\rho^{\prime}, \tau^{\prime}$ tend to 1 for $t \rightarrow 0$ ). Thus by the above argument, there exists a midpoint $y_{1}^{\prime}$ of $y_{0}^{\prime}$ and $y_{2}^{\prime}$ with $\left|p y_{1}^{\prime}\right|<s_{0}$. Clearly $y_{1}^{\prime}$ is also a midpoint of $y_{0}$ and $y_{2}$, and we are done.

\section{Normed VeCtor SPACES}

We recall some facts about normed vector spaces which we will use in the sequel.

Let $(V,\|\cdot\|)$ be a normed vector space. A line in $V$ is a map $c: \mathbb{R} \rightarrow V$ of the form $c(t)=a+t b$, where $b \neq 0$. A line is always a geodesic; however, if the normball is not strictly convex, there are other geodesics. We call a subset $C \subset V$ line-convex if for $x, y \in C$ the segment $[0,1] \rightarrow V, t \mapsto t y+(1-t) x$ is also contained in $C$. We use the notion line-convex since it differs from the notion of convexity as defined in Section 2 for normed spaces with nonstrictly (line)-convex normball.

Let $\gamma \in \operatorname{ISO}(V)$. Then a line $c: \mathbb{R} \rightarrow V$ parameterized by arclength is called an axis of $\gamma$ if $\gamma c(t)=c(t+L)$ for some $L>0$. If $\gamma$ has an axis, then we denote with $\operatorname{Ax}(\gamma) \subset V$ the set of points, which lie on axes of $\gamma$. Then $\operatorname{Ax}(\gamma)$ is a closed, line-convex subset of $V$. If $\alpha \in \operatorname{ISO}(X)$ commutes with $\gamma$, then $\operatorname{Ax}(\gamma)$ is $\alpha$-invariant.

Essential for our argument is the following result of Schoenberg [Sch].

Theorem 3.1. A normed vector space $(V,\|\cdot\|)$ is a Ptolemy space if and only if it is an inner product space.

Using asymptotic cones, it is not difficult to prove

Lemma 3.2. If a normed vector space is roughly isometric to a Ptolemy space, then it is a Ptolemy space.

Therefore we obtain

Corollary 3.3. If a normed vector space $(V,\|\cdot\|)$ is roughly isometric to a Ptolemy space, then it is an inner product space.

\section{Busemann functions}

In this section $X$ always denotes a geodesic Ptolemy space. Let $c:[0, \infty) \rightarrow X$ be a geodesic ray parameterized by arclength. As usual we define the Busemann function $b_{c}(x)=\lim _{t \rightarrow \infty}(|x c(t)|-t)$. Since $b_{c}$ is the limit of the convex functions $d_{c(t)}-t$, it is convex.

The following proposition implies that, in a Ptolemy space, rays with sublinear distance to each other define (up to a constant) the same Busemann functions.

Proposition 4.1. Let $X$ be a Ptolemy space, let $c_{1}, c_{2}:[0, \infty) \rightarrow X$ be rays with Busemann functions $b_{i}:=b_{c_{i}}$, and assume $\lim _{t \rightarrow \infty} \frac{1}{t}\left|c_{1}(t) c_{2}(t)\right|=0$. Then $\left(b_{1}-b_{2}\right)$ is constant.

Proof. Let $p, q \in X$ and let $P_{i}(t)=\left|p c_{i}(t)\right|, Q_{i}(t)=\left|q c_{i}(t)\right|$. We define $P_{i}^{\prime}(t), Q_{i}^{\prime}(t)$, such that $P_{i}(t)=t+P_{i}^{\prime}(t)$ and $Q_{i}(t)=t+Q_{i}^{\prime}(t)$. Hence we have $b_{i}(p)=$ $\lim P_{i}^{\prime}(t)$ and $b_{i}(q)=\lim Q_{i}^{\prime}(t)$. Since $X$ is Ptolemy, the three numbers $P_{1}(t) Q_{2}(t)$, $P_{2}(t) Q_{1}(t),\left|c_{1}(t) c_{2}(t)\right||p q|$ satisfy the triangle inequality. The assumption

$$
\lim _{t \rightarrow \infty} \frac{1}{t}\left|c_{1}(t) c_{2}(t)\right|=0
$$


now implies

$$
\lim _{t \rightarrow \infty} \frac{1}{t}\left(P_{1}(t) Q_{2}(t)-P_{2}(t) Q_{1}(t)\right)=0
$$

and hence

$$
\lim _{t \rightarrow \infty}\left(P_{1}^{\prime}(t)+Q_{2}^{\prime}(t)-P_{2}^{\prime}(t)-Q_{1}^{\prime}(t)\right)=0 .
$$

The last limit implies $\left(b_{1}-b_{2}\right)(p)=\left(b_{1}-b_{2}\right)(q)$.

Now let $c: \mathbb{R} \rightarrow X$ be a geodesic line parameterized by arclength. Let $c^{ \pm}$: $[0, \infty) \rightarrow X$ be the rays $c^{+}(t)=c(t)$ and $c^{-}(t)=c(-t)$. Further let $b^{ \pm}:=b_{c^{ \pm}}$.

The following follows easily.

Lemma 4.2. $\left(b^{+}+b^{-}\right) \geq 0$ and $\left(b^{+}+b^{-}\right)=0$ on the line $c$.

We now consider these Busemann functions for lines with sublinear distance.

Proposition 4.3. Let $c_{1}, c_{2}: \mathbb{R} \rightarrow X$ be lines with $\lim _{t \rightarrow \infty} \frac{1}{t}\left|c_{1}(t) c_{2}(t)\right|=0$ and $\lim _{t \rightarrow \infty} \frac{1}{t}\left|c_{1}(-t) c_{2}(-t)\right|=0$. Then $\left(b_{1}^{+}+b_{1}^{-}\right)=\left(b_{2}^{+}+b_{2}^{-}\right)$.

Proof. By Proposition 4.1 we have that $\left(b_{1}^{+}-b_{2}^{+}\right)$and $\left(b_{1}^{-}-b_{2}^{-}\right)$are constant, which implies that $\left(b_{1}^{+}+b_{1}^{-}-b_{2}^{+}-b_{2}^{-}\right)=a$ for some constant $a$. We show that $a=0$. If $p_{1} \in c_{1}(\mathbb{R})$, then by Lemma 4.2. $\left(b_{1}^{+}+b_{1}^{-}\right)\left(p_{1}\right)=0$ and $\left(b_{2}^{+}+b_{2}^{-}\right)\left(p_{1}\right) \geq 0$. Thus $a \leq 0$. If $p_{2} \in c_{2}(\mathbb{R})$, then $\left(b_{2}^{+}+b_{2}^{-}\right)\left(p_{2}\right)=0$ and $\left(b_{1}^{+}+b_{1}^{-}\right)\left(p_{2}\right) \geq 0$. Thus $a \geq 0$.

\section{Groups operating on $X$}

In this section $X$ always denotes a geodesic Ptolemy metric space. Let $\Gamma \subset$ $\operatorname{ISO}(X)$ be a subgroup of the isometry group operating properly and cocompactly on $X$. As already stated in the introduction, this implies that $X$ is proper and in particular complete. An element $\gamma \in \Gamma$ is a torsion element if $\gamma^{m}=$ id for some $m \geq 1$. To an element $\gamma \in \Gamma$ we associate the displacement function $d_{\gamma}(x):=|x \gamma x|$. A geodesic line is called an axis of an element $\gamma$ if there exists some $L>0$ such that $\gamma c(t)=c(t+L)$ for all $t \in \mathbb{R}$. We always parameterize lines by arclength. By $\operatorname{Ax}(\gamma):=\{x \in X \mid x$ lies on an axis of $\gamma\}$ we denote the union of all axes of $\gamma$.

Lemma 5.1. (1) $d_{\gamma}$ assumes the minimum $\min d_{\gamma} \geq 0$.

(2) $\min d_{\gamma}=0$ if and only if $\gamma$ is a torsion element.

(3) If $\gamma$ is not torsion, then $\operatorname{Ax}(\gamma)=\left\{x \in X \mid d_{\gamma}(x)\right.$ is minimal $\}$.

(4) If $c_{1}, c_{2}$ are axes of $\gamma$, then $\left|c_{1}(t) c_{2}(t)\right|$ is bounded.

(5) If $\alpha$ commutes with $\gamma$ and $c$ is an axis of $\gamma$, then also $\alpha c$ is an axis of $\gamma$.

Proof. (1) is standard. See e.g. the proof of Lemma 2.1 in [CS].

(2) If $\min d_{\gamma}=0$, then $\gamma$ has a fixed point. Since $\Gamma$ operates properly, $\gamma$ is a torsion element. If $\gamma$ is torsion, then, for given $x \in X$, the orbit $K=\left\{\gamma^{m}(x) \mid m \in \mathbb{Z}\right\}$ is a $\gamma$-invariant compact set. By Corollary 2.4, there exists a unique $p \in X$ such that $K \subset B_{r}(p)$, where $r$ is minimal. Then $p$ is fixed by $\gamma$; hence $\min d_{\gamma}=0$.

(3) Let $\gamma$ be a nontorsion element. Let $L=\min d_{\gamma}>0$ be the minimum of the displacement function. Let $x \in X$ with $d_{\gamma}(x)=L$. Let $c: \mathbb{R} \rightarrow X$ be the piecewise geodesic with $c(n L)=\gamma^{n}(x)$. Note that $c\left(\frac{2 n+1}{2} L\right)=m\left(\gamma^{n}(x), \gamma^{n+1}(x)\right)$ and hence $\left.\gamma c\left(\left(\frac{2 n-1}{2}\right) L\right)=c\left(\left(\frac{2 n+1}{2}\right) L\right)\right)$. Thus

$$
d_{\gamma}\left(c\left(\left(\frac{2 n-1}{2}\right) L\right)\right) \leq\left|c\left(\frac{2 n-1}{2} L\right) c(n L)\right|+\left|c(n L) c\left(\frac{2 n+1}{2} L\right)\right|=L .
$$


By the minimality of $L$ we have equality, which implies that $c_{\mid\left[\frac{2 n-1}{2} L \frac{2 n+1}{2} L\right]}$ is minimizing. Thus since $c_{\mid[n L,(n+1) L]}$ is also minimizing, $c$ is locally minimizing and hence a geodesic by Lemma 2.2. Thus $c$ is an axis of $\gamma$.

Assume now that $c: \mathbb{R} \rightarrow X$ is an axis with $\gamma c(t)=c(t+L), L>0$. We show that $L=\min d_{\gamma}$. Therefore let $x \in X$ and let $a=d(x, c(0))$. By the triangle inequality,

$$
m L=\left|c(0) \gamma^{m} c(0)\right| \leq|c(0) x|+\left|x \gamma^{m} x\right|+\left|\gamma^{m} x \gamma^{m} c(0)\right| \leq a+m d_{\gamma}(x)+a .
$$

Hence $m L \leq 2 a+m d_{\gamma}(x)$ for all $m$, which implies $d_{\gamma}(x) \geq L$.

(4) Since $c_{1}, c_{2}$ are axes parameterized by arclength, we have $\left|c_{1}(t+L) c_{2}(t+L)\right|=$ $\left|c_{1}(t) c_{2}(t)\right|$.

(5) If $\alpha$ commutes with $\gamma$ and $\gamma c(t)=c(t+L)$, then $\gamma(\alpha c(t))=\alpha(\gamma c(t))=$ $\alpha c(t+L)$; hence $\alpha \circ c$ is also an axis of $\gamma$.

Now let $\gamma$ be a nontorsion element of $\Gamma$. We define $b_{\gamma}=b_{c}$, where $c$ is an axis of $\gamma$. Since two axes have bounded distance, $b_{\gamma}$ is well defined up to a constant by Proposition 4.3. More generally we define $b_{\gamma}^{ \pm}=b_{c}^{ \pm}$and $B_{\gamma}=b_{\gamma}^{+}+b_{\gamma}^{-}$. Note that $B_{\gamma}$ is well defined and does not depend on the choice of an axis by Proposition 4.1

Lemma 5.2. (1) If $\alpha$ commutes with $\gamma$, then $b_{\gamma}(\alpha(x))-b_{\gamma}(x)$ is constant (in $\left.x\right)$.

(2) $b_{\gamma}(\gamma(x))-b_{\gamma}(x)=\min d_{\gamma}$.

(3) $B_{\gamma}=0$ on all axes of $\gamma$.

(4) $B_{\gamma^{-1}}=B_{\gamma}$ and $B_{\gamma}(\alpha(x))=B_{\alpha^{-1} \gamma \alpha}(x)$ for all $\alpha \in \Gamma$.

Proof. (1) If $\alpha$ commutes with $\gamma$ and $c: \mathbb{R} \rightarrow X$ is an axis of $\gamma$, then $\alpha^{-1} \circ c$ is also an axis of $\gamma$ and hence in bounded distance to $c$. Thus $b_{\alpha^{-1} \circ c}$ and $b_{c}$ differ by a constant by Proposition 4.1. Since $b_{c}(\alpha x)=b_{\alpha^{-1} \text { oc }}(x)$ we obtain the result.

(2) Because of (1) we need to compute the equality only for $x=c(0)$, where $c$ is an axis of $\gamma$. Since $\gamma c(0)=c(L)$ with $L=\min d_{\gamma}$ we are done.

(3) is merely a reformulation of Lemma 4.2 .

(4) follows from an easy computation.

Now the proof of Theorem 1.3 follows from the arguments in Section III of [CS]. The essential tool for the proof in that paper was Corollary 2.6, which corresponds exactly to our Lemma 5.2. One can easily check that the proof in the cited paper transfers word by word to the present situation.

\section{Affine functions}

Let $X$ be a geodesic metric space. Recall that for $x, y \in X$ we denote by $m(x, y)=\left\{z \in X \mid d(x, z)=d(z, y)=\frac{1}{2} d(x, y)\right\}$ the set of midpoints of $x$ and $y$. A map $f: X \rightarrow Y$ between two geodesic metric spaces is called affine if for all $x, y \in X$ we have $f(m(x, y)) \subset m(f(x), f(y))$. Thus a map is affine if and only if it maps geodesics parameterized proportionally to arclength into geodesics parameterized proportionally to arclength. An affine map $f: X \rightarrow \mathbb{R}$ is called an affine function. Note that this definition coincides with the definition given in Section 2 . For $X$ and $Y$ line convex subsets of linear spaces, call $f: X \longrightarrow Y$ line affine if for any $x, z \in X, f\left(\frac{1}{2} x+\frac{1}{2} z\right)=\frac{1}{2} f(x)+\frac{1}{2} f(z)$. Notice that an affine function which takes line segments to line segments is line affine.

Given a geodesic metric space $X$, we set

$$
\mathcal{A}^{\prime}(X):=\{f: X \rightarrow \mathbb{R} \mid f \text { affine and Lipschitz }\} .
$$


$\mathcal{A}^{\prime}(X)$ is a Banach space, where $\|f\|$ is the optimal Lipschitz constant. We set $\mathcal{A}(X):=\mathcal{A}^{\prime}(X) / \sim$, where $f \sim g$ if $(f-g)$ is constant. By $[f]$ we denote the equivalence class of $f$. Then also $\mathcal{A}(X)$ is a Banach space, where $\|[f]\|=\|f\|$. The group $\operatorname{ISO}(X)$ acts on $\mathcal{A}^{\prime}(X)$ (and $\mathcal{A}(X)$ ) by linear isometries via $\gamma(f)(x)=$ $f\left(\gamma^{-1}(x)\right)$ (resp. $\left.\gamma([f])=[\gamma(f)]\right)$. Let $\mathcal{A}^{*}(X)$ be the Banach dual space of $\mathcal{A}(X)$ with the norm

$$
\|\rho\|=\sup _{[f] \in \mathcal{A}(X)} \frac{|\rho([f])|}{\|[f]\|} .
$$

Also $\operatorname{ISO}(X)$ operates linearly on $\mathcal{A}^{*}$ by isometries via $\gamma(\rho)([f])=\rho\left(\gamma^{-1}([f])\right)$. For $x, y \in X$ let $E(x, y) \in \mathcal{A}^{*}(X)$ be the evaluation map $E(x, y)([f])=f(x)-f(y)$. It follows directly from the definitions that $\|E(x, y)\| \leq|x y|$. For $\gamma \in \operatorname{ISO}(X)$ we have $\|E(\gamma(x), \gamma(y))\|=\|E(x, y)\|$.

For a given basepoint $o \in X$ consider the subset $\mathcal{E}_{o}=\{E(x, o) \mid x \in X\} \subset \mathcal{A}^{*}$ and the map

$$
A_{o}: X \rightarrow \mathcal{E}_{o}, \quad x \mapsto E(x, o) .
$$

If $x, y \in X$ and $z \in m(x, y)$, then for all $[f] \in \mathcal{A}$ we have

$$
E(z, o)[f]=f(z)-f(o)=\frac{1}{2}(f(x)+f(y))-f(o)=\left(\frac{1}{2} E(x, o)+\frac{1}{2} E(y, o)\right)[f] .
$$

Thus $A_{o}$ is affine and maps the geodesics in $X$ to lines in $\mathcal{E}_{o} \subset \mathcal{A}^{*}$. In particular $\mathcal{E}_{o}$ is a line-convex subset of $\mathcal{A}^{*}$. Since $\|E(x, o)-E(y, o)\|=\|E(x, y)\| \leq|x y|$ we see that $A_{o}$ is 1 -Lipschitz.

We consider the map $a_{o}: \operatorname{ISO}(X) \rightarrow \operatorname{ISO}\left(\mathcal{E}_{o}\right)$ given by $a_{o}(\gamma)(E(x, o)):=$ $E(\gamma x, o)$. Indeed $a_{0}(\gamma)$ is an affine isometric operation, since

$$
\|E(\gamma x, o)-E(\gamma y, o)\|=\|E(\gamma x, \gamma y)\|=\|E(x, y)\|=\|E(x, o)-E(y, o)\| .
$$

With respect to this affine operation the map $A_{o}$ is $\operatorname{ISO}(X)$-equivariant, i.e. $A_{o}(\gamma x)$ $=a_{o}(\gamma) A_{o}(x)$. The isometries $a_{o}(\gamma)$ map lines in $\mathcal{E}_{o}$ to lines in $\mathcal{E}_{o}$, so $a_{o}(\gamma)$ is line affine. We remark that this affine action of $\operatorname{ISO}(X)$ on $\mathcal{E}_{o}$ is not the restriction of the linear action of $\operatorname{ISO}(X)$ on $\mathcal{A}^{*}$ to the subset $\mathcal{E}_{o}$. Also notice that if $U$ is a line convex subset of $\mathcal{E}_{o}$, then $A_{o}^{-1}(U)$ is convex.

We recollect all this information in the following.

Lemma 6.1. If $X$ is a geodesic metric space, then the map $A_{o}: X \rightarrow \mathcal{E}_{o}$ is a surjective, affine, 1-Lipschitz and $\operatorname{ISO}(X)$-equivariant map of $X$ onto the lineconvex subset $\mathcal{E}_{o} \subset \mathcal{A}^{*}$.

Definition 6.2. We say that affine functions separate points on $X$ if for different $x, y \in X$ there exists $f \in \mathcal{A}^{\prime}(X)$ with $f(x) \neq f(y)$.

Note that affine functions separate points if and only if the map $A_{o}$ is injective. In this case we have the following strong result of Hitzelberger and Lytchak HL.

Theorem 6.3. If affine functions separate points, then $A_{o}: X \rightarrow \mathcal{E}_{o}$ is an isometry.

\section{Proof of Theorems 1.1, 1.2 and Corollary 1.4}

In this section we assume that $X$ is a geodesic Ptolemy space and $\Gamma \subset \operatorname{ISO}(X)$ is a crystallographic group operating properly and cocompactly on $X$. Recall that under these assumptions $X$ is proper.

Recall the following characterization of crystallographic groups (see [W], Theorem 3.2.9). 
A group $\Gamma$ is crystallographic if and only if $\Gamma$ has a normal, free abelian subgroup $\Gamma^{*}$ of finite rank and finite index in $\Gamma$ which is maximal abelian in $\Gamma$. In that case, $\Gamma^{*}$ is unique.

Note that $\Gamma^{*} \simeq \mathbb{Z}^{n}$, since $\Gamma^{*}$ is free of finite rank. Since $\Gamma^{*}$ has finite index, $\Gamma^{*}$ also operates cocompactly on $X$. Let $\alpha \in \Gamma^{*} \backslash$ id, let $c$ be an axis of $\alpha$ and let $b_{\alpha}^{ \pm}=b_{c}^{ \pm}$be the corresponding Busemann functions. It follows from Lemma 5.2 that the function $B_{\alpha}=b_{\alpha}^{+}+b_{\alpha}^{-}$is $\Gamma^{*}$ invariant. Since $\Gamma^{*}$ acts cocompactly the function $B_{\alpha}$ is bounded.

Proof of Theorem 1.2. We now assume in addition that $X$ is a manifold. Thus by Corollary 2.8 all geodesics in $X$ can be extended. Thus the bounded convex functions $B_{\alpha}$ are constant for all $\alpha \in \Gamma^{*} \backslash$ id. Since $B_{\alpha}(x)=0$ for a point on an axis of $\alpha$, we have that $B_{\alpha}=0$ on $X$. Thus $B_{\alpha}=b_{\alpha}^{+}+b_{\alpha}^{-}$is in particular affine, and since the $b_{\alpha}^{ \pm}$are convex, the functions $b_{\alpha}^{ \pm}$are actually affine.

Since $A_{o}$ maps geodesics in $X$ to lines in $\mathcal{E}_{o}$ and since all geodesics are extendable, the image $\mathcal{E}_{o}$ is an affine subspace of $\mathcal{A}^{*}$.

We will use the following fact which follows immediately from the cocompactness of $\Gamma^{*}$ : there exists a constant $C_{1}>0$ such that for all $p, q \in X$ there exists $\alpha \in \Gamma^{*}$ with $|p \alpha(q)| \leq C_{1}$.

We now show that the affine functions separate points. Therefore let $x, y \in X$ with $x \neq y$. We first assume that $|x y|>6 C_{1}$. Then there exists $\alpha \in \Gamma^{*} \backslash$ id such that $|\alpha(x) y| \leq C_{1}$. There also exists an axis $c: \mathbb{R} \rightarrow X$ of $\alpha$ such that $|x c(0)| \leq C_{1}$ (this follows since for any axis $c^{\prime}$ of $\alpha$ and any $\gamma \in \Gamma^{*}$, also $\gamma \circ c^{\prime}$ is an axis of $\alpha$ ). Consider now the affine function $b_{\alpha}$. Since $b_{\alpha}$ is 1-Lipschitz, we have $\left|b_{\alpha}(x)-b_{\alpha}(c(0))\right| \leq C_{1},\left|b_{\alpha}(\alpha c(0))-b_{\alpha}(\alpha x)\right| \leq C_{1},\left|b_{\alpha}(\alpha x)-b_{\alpha}(y)\right| \leq C_{1}$, and $\left|b_{\alpha}(\alpha c(0)) b_{\alpha}(c(0))\right|=|c(0) \alpha(c(0))|$ by Lemma 5.2 (2). Thus

$$
\left|b_{\alpha}(x)-b_{\alpha}(y)\right| \geq|c(0) \alpha(c(0))|-3 C_{1} \geq|x y|-6 C_{1}>0,
$$

which shows that $x, y$ are separated by $b_{\alpha}$.

Now let $x, y \in X$ be arbitrary with $x \neq y$. Let $c: \mathbb{R} \rightarrow X$ be the unit speed geodesic with $c(0)=x$ and $c(\tau)=y$ for $\tau=|x y|$. Let $s>6 C_{1}$ and $y^{\prime}=c(s)$. By the above argument there exists an affine function $f$ with $f(x) \neq f\left(y^{\prime}\right)$. Thus the affine function $f \circ c$ is not constant, and hence $f(x) \neq f(y)$.

Since affine functions separate points, the map $A_{o}: X \rightarrow \mathcal{E}_{o}$ is an isometry by the Hitzelberger-Lytchak Theorem. Thus $\mathcal{E}_{o}$ is an affine subspace of the normed vector space $\mathcal{A}^{*}$ which is in addition a Ptolemy space. By Schoenberg's result it is an inner product space and hence isometric to the standard $\mathbb{R}^{n}$, where $n=\operatorname{dim} X$.

We now turn to the proof of Theorem 1.1. We assume that a crystallographic group operates properly and cocompactly on the geodesic Ptolemy space $X$.

Let $X^{\prime} \subset X$ be a nonempty, closed, convex and $\Gamma$-invariant subset, which is minimal with respect to these properties. Since $\Gamma$ operates cocompactly on $X$, it is easy to show that such a minimal set $X^{\prime}$ exists. Namely, consider an arbitrary chain $\left\{A_{j}\right\}_{j \in J}$ of such sets. Then this chain is bounded by the nonempty, closed, convex and $\Gamma$-invariant set $\bigcap A_{j}$. In order to see that this set is nonempty, just take a compact, fundamental region $K$ of $\Gamma$ and consider the intersections $A_{j} \cap K$, $j \in J$. Since $K$ is a compact fundamental region, and the $A_{j}$ are $\Gamma$-invariant, it follows that $\bigcap A_{j} \neq \emptyset$. Now the existence of a minimal set $X^{\prime}$ follows from Zorn's Lemma. 
By Corollary 2.5 there exists a continuous projection $\pi: X \rightarrow X^{\prime}$. This projection is $\Gamma$-equivariant and since $\Gamma$ operates cocompactly on $X$ as well as on $X^{\prime}$, there exists a constant $C \geq 0$ such that $|x \pi(x)| \leq C$ for all $x \in X$ and hence $\pi$ is a rough isometry. Thus we have to prove the theorem only for $X^{\prime}$. Thus we can suppose that $X$ satisfies the following additional

Assumption. If $W \subset X$ is a nonempty closed convex and $\Gamma$-invariant subset, then $W=X$.

Lemma 7.1. For all $x \in X$ and for all $\alpha \in \Gamma^{*} \backslash$ id we have

(1) $B_{\alpha}(x)=0$ and hence the functions $b_{\alpha}^{ \pm}$are affine,

(2) $A_{o}(x) \in \operatorname{Ax}\left(a_{o}(\alpha)\right)$.

Proof. Let $W \subset X$ be the subset of all $x \in X$ such that (1) and (2) hold for all $\alpha \in \Gamma^{*} \backslash$ id. We have to show that $W=X$.

Since $B_{\alpha}$ is a continuous convex function, the condition (1) is closed and convex. Since the action of $\operatorname{ISO}(X)$ on $\mathcal{E}_{o}$ is line-convex, $\operatorname{Ax}\left(a_{o}(\alpha)\right)$ is line-convex and of course closed. Thus $A_{o}^{-1}\left(\operatorname{Ax}\left(a_{o}(\alpha)\right)\right.$ is a closed convex subset of $X$.

Since $\Gamma^{*}$ is a normal subgroup of $\Gamma,(1)$ and (2) describe a $\Gamma$-invariant condition. Thus $W$ is closed convex and $\Gamma$-invariant.

We now show that $W$ is not empty. To prove this, let $W^{\prime} \subset X$ be a nonempty, closed, convex and $\Gamma^{*}$-invariant subset, which is minimal with these properties. Since $\Gamma^{*}$ operates (as a finite index subgroup of $\Gamma$ ) cocompactly on $X$, it follows as above that such a minimal set $W^{\prime}$ exists. We show that $W^{\prime} \subset W$ and hence $W$ is not empty. Let $\alpha \in \Gamma^{*} \backslash$ id. Since $\Gamma^{*}$ is abelian, the convex function $B_{\alpha}$ is $\Gamma^{*}$-invariant and hence $\left\{B_{\alpha}=0\right\}$ is closed, convex and $\Gamma^{*}$-invariant.

Now the function $d_{\alpha}$ assumes a minumum on the subset $W^{\prime}$; hence there exists an axis $c: \mathbb{R} \rightarrow W^{\prime}$ of $\alpha$.

Thus it follows from Lemma 5.2 (3) that $W^{\prime} \cap\left\{B_{\alpha}=0\right\} \neq \emptyset$. This shows that $W^{\prime} \subset\left\{B_{\alpha}=0\right\}$, since $W^{\prime}$ was assumed to be minimal and $\left\{B_{\alpha}=0\right\}$ is closed, convex and $\Gamma^{*}$-invariant.

Recall that $A_{o}$ maps geodesics to lines or to a point. We show that $A_{o} \circ c(t)=$ $E(c(t), o)$ is actually a complete line. Since $B_{\alpha}$ is constant and hence affine, also the functions $b_{\alpha}^{ \pm}$are affine. Since $b_{\alpha}^{ \pm}$are not constant on $c(t)$, the map $t \mapsto E(c(t), o)$ is also not constant. It follows that $A_{o} \circ c$ is a complete line which is $a_{o}(\alpha)$-invariant and hence an axis of $a_{o}(\alpha)$. Since $\Gamma^{*}$ is abelian, the set $C:=\operatorname{Ax}\left(a_{o}(\alpha)\right) \subset \mathcal{E}_{o}$ is closed, line-convex and $a_{o}\left(\Gamma^{*}\right)$-invariant. Hence $A_{o}^{-1}(C)$ is closed, convex and $\Gamma^{*}$-invariant. By minimality $W^{\prime} \subset A_{o}^{-1}(C)$, which implies that for $x \in W^{\prime}$, we have $A_{o}(x) \in \operatorname{Ax}\left(a_{o}(\alpha)\right)$. Thus we have shown that $W^{\prime} \subset W$ and hence $W \neq \emptyset$. By the additional assumption, $W=X$.

Lemma 7.2. The map $A_{o}: x \mapsto E(x, o)$ is a rough isometry.

Proof. We know already that $A_{o}$ is 1-Lipschitz.

We use arguments which are very similar to the arguments in the proof of Theorem 1.2 Again $\Gamma^{*}$ operates cocompactly on $X$, and hence there exists $C_{1}>0$ such that for all $p, q \in X$ there exists $\alpha \in \Gamma^{*}$ with $|p \alpha(q)| \leq C_{1}$.

Now let $x, y \in X$ with $|x y|>6 C_{1}$, and choose $\alpha \in \Gamma^{*}$ exactly as in the proof of Theorem 1.2 , 
Then

$$
\|E(x, o)-E(y, o)\|=\| E\left(x, y\|\geq\| E(x, y)\left(\left[b_{\alpha}\right]\right) \|=\left|b_{\alpha}(x)-b_{\alpha}(y)\right| \geq|x y|-6 C_{1},\right.
$$

where the arguments are exactly as above. The claim follows.

Lemma 7.3. $\mathcal{E}_{o}$ is an affine subspace of $\mathcal{A}^{*}$ with $\operatorname{dim}\left(\mathcal{E}_{o}\right)=\operatorname{rank}\left(\Gamma^{*}\right)$.

Proof. By the above lemma all $a_{o}(\alpha), \alpha \in \Gamma^{*} \backslash$ id operate as translations on the set $\mathcal{E}_{o}$. Thus the line-convex hull of $a_{0}\left(\Gamma^{*}\right) E(x, o)$ is an affine subspace $H_{x} \subset \mathcal{E}_{o}$ of dimension equal to $\operatorname{rank}\left(\Gamma^{*}\right)$ for all $x \in X$. Note that all $H_{x}$ are parallel to each other. Since $\Gamma^{*}$ is a finite index normal subgroup of $\Gamma$. there are finitely many points $x_{0}, \ldots, x_{k} \in X$ such that $\Gamma$ leaves the set $H_{x_{o}}, \ldots, H_{x_{k}}$ invariant. The line-convex hull of these subspaces defines a finite dimensional affine subspace $V$ of $\mathcal{A}^{*}$, and the group $a_{o}(\Gamma)$ leaves this space invariant and operates affinely on $V$, i.e. maps lines to lines. Since $a_{o}(\Gamma)$ also maps parallels of $H_{x}$ to parallels of $H_{x}$, $a_{o}(\Gamma)$ also operates affinely on the quotient space $V / H_{x_{o}}$, and has on that space a finite orbit $\left[x_{0}\right], \ldots,\left[x_{k}\right]$. Therefore this operation has a fixed point $[v]$ which is contained in the line-convex hull of $\left[x_{0}\right], \ldots,\left[x_{k}\right]$. This implies that there exists an affine subspace $H$ parallel to $H_{x_{o}}$ which is $a_{o}(\Gamma)$-invariant and which is contained in the line-convex hull of $H_{x_{o}}, \ldots, H_{x_{k}}$. Thus $H$ is in the image of $A_{o}$.

Let $W=A_{o}^{-1}(H)$. Then $W \subset X$ is a nonempty convex $\Gamma$-invariant subset, and hence $W=X$ by our additional assumption. It follows that for all $x \in X, H_{x} \subset H$ and by dimension reasons $H_{x}=H$. It follows that $\mathcal{E}_{o}=H$, which proves the lemma.

Thus we see that $A_{o}: X \rightarrow \mathcal{E}_{o}$ is a $\Gamma$-equivariant rough isometry. By Lemma 7.3. $\mathcal{E}_{o}$ is a normed vector space which is roughly isometric to the Ptolemy space $X$. By the Corollary 3.3 to Schoenberg's result, we see that $\mathcal{E}_{o}$ is an inner product space and hence we obtain Theorem 1.1.

Finally we prove Corollary 1.4 .

Let $\Gamma$ be a virtual polycyclic group. Let $\Pi \subset \Gamma$ be a polycyclic subgroup of finite index. By a theorem of Hirsch (see e.g. [R], p. 139), $\Pi$ contains a torsion-free solvable subgroup $\Sigma$ of finite index. Then also $\Sigma$ operates cocompactly on $X$ and by Theorem 1.3 all abelian subgroups of $\Sigma$ are straight. Note further that all abelian subgroups of $\Sigma$ are finitely generated, since $\Pi$ is polycyclic. Now a torsion-free solvable group such that all abelian subgroups are finitely generated and straight is a crystallographic group by [CS], Lemma 4.3. Thus $\Sigma$ and hence also $\Gamma$ contains a free abelian subgroup $\Sigma^{*}$ of finite index and finite rank. It follows that $\Gamma$ also has a normal free abelian subgroup $\Gamma^{*}$ of finite index and finite rank. But this fact is the only property of $\Gamma$ which we needed in the proof of Theorems 1.1 and 1.2 ,

At the end we pose an

Open question. Let $\Gamma$ be a crystallographic group operating properly and cocompactly on a geodesic Ptolemy space $X$. Does there exist a totally geodesic flat Euclidean subspace $F \subset X$ which is $\Gamma$-invariant?

\section{ACKNOWLEDGMENT}

It is a pleasure to thank Alexander Lytchak for many inspiring discussions as well as the referee for his careful reading of the manuscript and useful comments. 


\section{REFERENCES}

[B] M. Bourdon, Sur le birapport au bord des CAT(-1)-espaces, Inst. Hautes Études Sci. Publ. Math. No. 83 (1996), 95-104. MR1423021 (97k:58123)

[BH] M. Bridson and A. Haefliger, Metric spaces of non-positive curvature, Grundlehren der Mathematischen Wissenschaften 319, Springer-Verlag, Berlin, 1999. xxii+643 pp. MR 1744486 (2000k:53038)

[BFW] S.M. Buckley, K. Falk and D.J. Wraith, Ptolemaic and CAT(0), Glasg. Math. J. 51 (2009), no. 2, 301-314. MR 2500753 (2010e:53070)

$[\mathrm{Bu}]$ D. Burago, Periodic Metrics, Adv. Soviet Math. 9 (1992), 205-210. MR.1166203 (93c:53029)

[BuI] D. Burago and S. Ivanov, Riemannian tori without conjugate points, Geom. Funct. Anal. 4 no. 3 (1994), 259-269. MR.1274115 (95h:53049)

[CS] Ch. Croke and V. Schroeder, The fundamental group of compact manifolds without conjugate points, Comment. Math. Helvetici 61 (1986), 161-175. Free available via http://www.math.ch/swissdml/. MR847526 (87i:53060)

[FLS] Th. Foertsch, A. Lytchak and V. Schroeder, Nonpositive curvature and the Ptolemy inequality, Int. Math. Res. Not. IMRN 2007, no. 22, 15 pp. MR2376212 (2009d:53049a)

[FS] Th. Foertsch and V. Schroeder, Hyperbolicity, CAT(-1)-spaces and the Ptolemy Inequality, to appear in Math. Annalen.

[G] M. Gromov, Asymptotic invariants of infinite groups, Geometric Group Theory, Proc. of the Symposium held in Susses 1991, Vol. 2, edited by A. Niblo and M.Roller, Cambridge Univ. Press, 1993. MR 1253544 (95m:20041)

[Ha] U. Hamenstädt, A new description of the Bowen-Margulis measure, Ergodic Theory Dynam. Systems 9 (1989), 455-464. MR1016663 (91b:58191)

[HL] P. Hitzelberger and A. Lytchak, Spaces with many affine functions, Proc. Amer. Math. Soc. 135, Number 7 (2007), 2263-2271. MR2299504 (2008g:53045)

[R] D. Robinson, Finiteness conditions and generalized soluble groups, Part 2, Springer, Berlin Heidelberg New York, 1972. MR0332990 (48:11315)

[Sch] I. J. Schoenberg, A remark on M. M. Day's characterization of inner-product spaces and a conjecture of L. M. Blumenthal, Proc. Amer. Math. Soc. 3 (1952), 961-964. MR0052035 $(14: 564 \mathrm{c})$

[W] J. Wolf, Spaces of constant curvature, McGraw-Hill, New York, 1967. MR 0217740 (36:829)

Mathematisches Institut, Universität Bonn, Beringstrasse 1, D-53115 Bonn, Germany

E-mail address: foertsch@math.uni-bonn.de

Institut für Mathematik, Universität Zürich, Winterthurer Strasse 190, CH-8057 ZÜRICH, SWITZERLAND

E-mail address: vschroed@math.unizh.ch 